\title{
The UKF Based Approach to Improving Attitude and Position of Quadcopter Through Autonomous and Non-Autonomous Flight
}

\author{
Ahmed Abdulmahdi Abdulkareem ${ }^{1}$, Basil H. Jasim*2, Safanah Mudheher Raafat ${ }^{3}$ \\ ${ }^{1}$ College of Science, Physics Department, University of Wasit, Wasit, Iraq \\ 2 Electrical Engineering Department, University of Basrah, Basra, Iraq \\ ${ }^{3}$ Control and System Engineering Department, University of Technology, Baghdad, Iraq
}

Correspondence

* Basil H Jasim

Electrical Engineering Department,

University of Basrah, Basra, Iraq

Email: hanbas632@gmail.com

\begin{abstract}
The gyroscope and accelerometer are the basic sensors used by most Unmanned Aerial Vehicle (UAV) like quadcopter to control itself. In this paper, the fault detection of measured angular and linear states by gyroscope and accelerometer sensors are present. Uncertainties in measurement and physical sensors itself are the main reasons that lead to generate noise and cause the fault in measured states. Most previous solutions are process angular or linear states to improving the performance of quadcopter. Also, in most of the previous solutions, KF and EKF filters are used, which are inefficient in dealing with high nonlinearity systems such as quadcopter. The proposed algorithm is developed by the robust nonlinear filter, Unscented Kalman Filter (UKF), as an angular and linear estimation filter. Simulation results show that the proposed algorithm is efficient to decrease the effect of sensors noise and estimate accurate angular and linear states. Also, improving the stability and performance properties of the quadcopter. In addition, the new algorithm leads to increasing the range of nonlinearity movements that quadcopter can perform it.

KEYWORDS: Linear state estimation of Quadcopter, Angular state estimation of Quadcopter, Controller Quadcopter, UKF, Noisy states measured by Gyroscope sensor, Noisy states measured by Accelerometer sensor.
\end{abstract}

\section{INTRODUCTION}

In last few years, the use of quadcopters has increased dramatically in the military and civilian fields due to their characteristics of takeoff and landing vertically as well as hovering. $[1,2]$. Some of these applications are require high stability during flight and hovering, such as examining the structures of high building, bridges, through special cameras to detect cracks early and treat them [3]. A highly efficient control system is needed by quadcopter that is used for these applications to increase its performance, which is one of the most important characteristics. The movements of quadcopter in $\mathrm{x}-\mathrm{y}$ plane is controlled through the angular position (attitude) around linear body axis $\left(\mathrm{x}_{\mathrm{B}}, \mathrm{y}_{\mathrm{B}}, \mathrm{z}_{\mathrm{B}}\right)$ of quadcopter. Angular position mean the rotation angles Roll, Pitch, and Yaw of quadcopter around the body frame as shown in Fig. 1[4, 5].

The angular velocity of quadcopter around body frame is measured by the gyroscope sensor while the linear acceleration toward the same frame is measured by accelerometer [6]. The attitude is calculated by integrated the measured angular velocity through mathematical algorithm.
While the linear position is calculated by double integrated the measured linear acceleration [7].

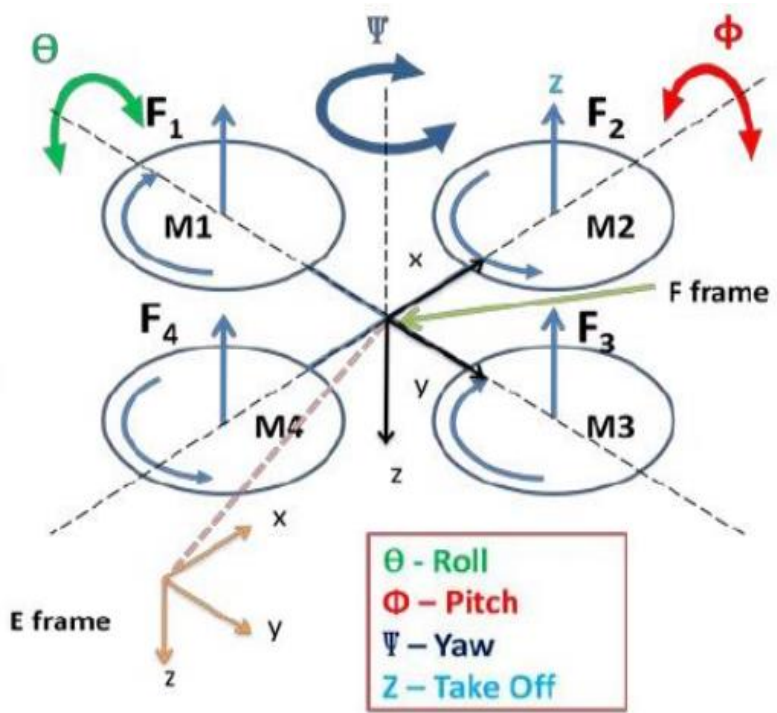

Fig. 1: The Body and Earth frames of quadcopter [4]

This is an open access article under the terms of the Creative Commons Attribution License, which permits use, distribution and reproduction in any medium, provided the original work is properly cited.

(C) 2020 The Authors. Published by Iraqi Journal for Electrical and Electronic Engineering by College of Engineering, University of Basrah. 
Most quadcopters are uses some of cheap sensors to measure all its states (angular and linear position, and altitude) because high precision sensors are very expensive and its price may exceed the price of quadcopter itself [8]. Therefore, the calculated angular and linear states have a noise generated by physical sensors and uncertainties in measurements [9]. The noise will decrease the robustness of controller and this lead to decrease the performance of quadcopter [10].

Recently, Different estimation filters are used to decrease the effect of noise and estimate more accurate states that is lead to increase the controller efficiency. The more popular used filters are Kalman Filter $(K F)$ and Extended Kalman Filter $(E K F)$ [11]. These filters have a drawback to operate properly with high nonlinearity systems because they need to linearize the nonlinear system through Jacobean matrix to estimate his states. So these filter will push the system out of control if high nonlinearity situation happen through his movements like quadcopter [12]. Most of the previous research focuses on using one of these filters to estimate more accurately linear or angular states of the quadcopter but these filters can't work properly with high nonlinearity situations as we mentioned earlier. Therefore, a robust and efficient nonlinear filter should be used to improving state estimation process. One of the most important real time nonlinear estimation filters is Unscented Kalman filter $(U K F)$ which will use it with our proposed algorithm in this paper.

The UKF is proposed to estimate accurate angular and linear states of quadcopter. The UKF is a robust modern nonlinear estimation filter which can operate properly with high nonlinearity system $[13,14]$. The proposed filter has precision larger than $\mathrm{KF}, \mathrm{EKF}$, and other old filters because it uses a robust estimation mechanism (Unscented Transform) and does not need to linearization operation [12]. Most of the previous research improved the performance of the quadcopter by improving the process of estimating linear or angular states. In this research, the process of estimating both linear and angle states will be improved at the same time through the use of an efficient and high-precision filter to deal with nonlinear systems.

\section{LITERATURE SURVEY}

In last few years, several methods are proposed to estimate accurate quadcopter states. The first last proposed methods which are used to estimate only linear states can be summarized as follows: In [8], this approach uses Kalman Filter (KF) to estimate actual linear states of quadcopter from noisy measured states. The EKF is an upgraded version of $\mathrm{KF}$ to better deal with nonlinear systems within a specified range of nonlinearity. The $\mathrm{KF}$ and EKF filters are uses the Jacobian Matrix method in the Linearization process to convert the nonlinear system to linear one. Then, they estimate more accurate states for linearized system. But these transformations can operate within certain limits of nonlinearity of system because they use some approximation arithmetic operations that may push the system out of control in high nonlinear conditions. In addition, the EKF is need to more iterations through estimation process and this make its performance relatively slower [15].

The KF is designed especially for linear systems while the quadcopter is nonlinear system. Therefore, this filter does not work properly in the nonlinear behavior of quadcopter and may push the quadcopter out of control, while in the approach of [10], the authors uses Extended Kalman Filter (EKF) to estimate actual linear states of quadcopter from noisy measured states. EKF is a modified version of KF designed to operate properly with nonlinear systems. The EKF uses linearized model of nonlinear system to estimate its states. Therefore, its stability is highly affected by nonlinearities of the system. Moreover, the EKF has a relatively slower performance since it takes more iterations to estimate actual states [15].

The second proposed methods which are used to estimate only angular states can be summarized as follows: in [16], the authors are use Smooth Sliding Mode (SSM) estimator to estimate the attitude of quadcopter under smoothness disturbance assumption. This method is tested under smoothness constraint only while the real quadcopter may expose to different disturbance. Also, the authors don't process the noisy linear position of quadcopter measured by IMU sensors. The approach of [17] is to introduces an Attitude Heading Reference System (AHRS) by using three Axis Attitude Determination (TRIAD) algorithm as the observation model and UKF as estimation filter. The authors don't process the noisy linear states. In this paper, the authors are present the fault estimation of UAV states caused by the sensors (Gyroscope and Accelerometer) of inertial measurements units (IMU). The sliding-mode observer and diagnostic algorithm are used in [18] to detect and process the bias fault of IMU. The authors do not access the noise generated by physical sensors and uncertainties in measurements of UAV states. Also, the proposed algorithms by authors doesn't process noisy linear states. In addition, the proposed algorithm can't operate properly with high nonlinearity systems [19]. In [11, 20], KF is used to estimate attitude (angular states) from noisy measured states by quadcopter sensors. As we mentioned earlier, KF maybe push the system out of control when high nonlinearity situation of the system is appearing. In [21], Multiplicative EKF (MEKF) and adaptive $\mathrm{H}_{\infty}$ filter is used to estimate accurate angular states (attitude) of quadcopter. The MEKF can't work properly with high nonlinearity system as mentioned earlier and it may push the system out of control.

\section{DYNAMIC MODEL OF QUADCOPTER}

There are several mathematical methods that can be used to derive the dynamic model of quadcopter. The dynamic model is needed by different researchers to implement and test their developed new ideas to improving the performance of quadcopter. The quadcopter has four motors installed at the end of his four arms as shown in Fig. 2. The first two identical motors 1 and 3 are rotate clockwise (CW) while the other two motors 2 and 4 are rotate counterclockwise $(\mathrm{CCW})$. There are several forces can be shown in Fig. 2 that affect the quadcopter. Some of forces are external like the 
gravity $(\mathrm{g})$ and some of other forces are generated by the propellers of quadcopter's motors like moment of inertia $\left(I_{m}\right)$ around each motors and thrust force $(\mathrm{T})$ as shown in Fig. 2. The generated forces by quadcopter give it ability to fly and flow the desired path to perform the desired missions [22, 23].

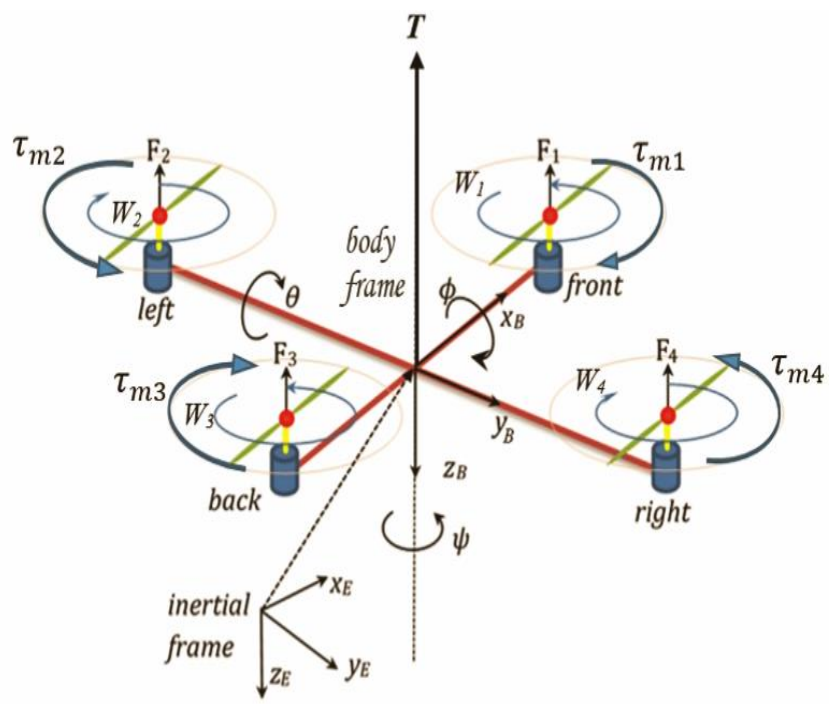

Fig.2: The Inertial and Body Frames of the quadcopter [28]

The mathematical model is very important because it give the researchers ability to perform and test their proposed algorithms on quadcopter through different simulation environments. So, the basic mathematical model equations that are used in our simulation will explain here.

$$
\left.\begin{array}{l}
\ddot{\phi}=\frac{I_{y}-I_{z}}{I_{x}} \dot{\theta} \dot{\psi}+\frac{U_{2}}{I_{x}} \\
\ddot{\theta}=\frac{I_{z}-I_{x}}{I_{y}} \dot{\phi} \dot{\psi}+\frac{U_{3}}{I_{y}} \\
\ddot{\psi}=\frac{I_{x}-I_{y}}{I_{z}} \dot{\theta} \dot{\phi}+\frac{U_{4}}{I_{z}}
\end{array}\right\}
$$

The variable $U_{2}, U_{3}$, and $U_{4}$ in equation 1 are represents the control signals generated by quadcopter's controller which are used by motors to generate required torques $\tau_{\phi}, \tau_{\ominus}$, and $\tau_{\psi}$ around linear axis [24, 27].

The linear motion of quadcopter in the $x-y$ plane and altitude (z), can be represented in terms of linear acceleration as in equation 2 .

$$
\left.\begin{array}{c}
\ddot{x}=\frac{U 1}{m}\left(c_{\psi} s_{\theta} c_{\phi}+s_{\psi} s_{\phi}\right) \\
\ddot{y}=\frac{U 1}{m}\left(s_{\psi} s_{\theta} c_{\phi}-c_{\psi} s_{\phi}\right) \\
\ddot{z}=\frac{U 1}{m} c_{\theta} c_{\phi}-g
\end{array}\right\}
$$

The $U_{l}$ variable is represent the control signal generated by the quadcopter's controller and it is responsible to generate required thrust force for quadcopter. The overall mass of quadcopter through flight is represented by the variable $m$.

where $U 1$ is the control input generated by flight controller to introduce the desired thrust force for quadcopter. $m$ represents mass of the quadcopter. $C_{\phi}=\cos (\phi), S_{\phi}=\sin (\phi)$, $\mathrm{C}_{\Theta}=\cos (\Theta), \mathrm{S}_{\Theta}=\sin (\Theta)$ [24-27]

Equations 1 and 2 can be used to test the new algorithms and methods proposed by researchers on the quadcopter by implementing them in a simulation program such as Matlab Simulink. By performing double integration for equations 1 and 2, all linear and angular states $(x, \dot{x}, y, \dot{y}, z, \dot{z}, \phi, \dot{\phi}, \theta, \dot{\theta}, \Psi, \dot{\psi})$

of quadcopter can be found and used to perform the simulation model of quadcopter.

Some applications like UKF need to use the state space of the system as state transition function to perform his algorithm. So, equation 3 is represent the state space of quadcopter that is programed and used in proposed algorithm [29].

$$
\left[\begin{array}{c}
\dot{x}_{1} \\
\dot{x}_{2} \\
\dot{x}_{3} \\
\dot{x}_{4} \\
\dot{x}_{5} \\
\dot{x}_{6} \\
\dot{x}_{7} \\
\dot{x}_{8} \\
\dot{x}_{9} \\
\dot{x}_{10} \\
\dot{x}_{11} \\
\dot{x}_{12}
\end{array}\right]=\left[\begin{array}{c}
x_{2} \\
(U 1 / m) *\left(\cos \left(x_{7}\right) \sin \left(x_{9}\right) \cos \left(x_{11}\right)+\sin \left(x_{7}\right) \sin \left(x_{11}\right)\right) \\
x_{4} \\
(U 1 / m) *\left(\cos \left(x_{7}\right) \sin \left(x_{9}\right) \sin \left(x_{11}\right)-\sin \left(x_{7}\right) \cos \left(x_{11}\right)\right) \\
x_{6} \\
\left((U 1 / m) *\left(\cos \left(x_{7}\right) \cos \left(x_{9}\right)\right)\right)-g \\
x_{8} \\
U 2 / I_{X X} \\
x_{10} \\
U 3 / I_{y y} \\
x_{12} \\
U 4 / I_{z Z}
\end{array}\right]
$$

Also, the measurement functions are representing the measured states $\left(X_{2}, X_{4}, X_{6}, X_{8}, X_{10}\right.$, and $\left.X_{12}\right)$ in the equation 3 and can be expressed as in equation (4):

$$
\left[\begin{array}{c}
x=\dot{x}_{1}=x_{2} \\
y=\dot{x}_{3}=x_{4} \\
z=\dot{x}_{5}=x_{6} \\
\phi=\dot{x}_{7}=x_{8} \\
\Theta=\dot{x}_{9}=x_{10} \\
\psi=\dot{x}_{11}=x_{12}
\end{array}\right]
$$

Equations (3) and (4) are used by UKF block to estimate accurate states of quadcopter.

\section{MODIFIED PID CONTROLLER}

The quadcopter is aerodynamically unstable and therefore a control system is needed to control its attitude and position to keep it stable during flight. The modified PID (PID) controller as shown in Fig. 3 is used in this paper because it is simple, efficient, and easy to implement in real and simulation platforms [30]. The modified PID controller is used to control the quadcopter through flight and hovering by decreasing the error between desired and measured states.

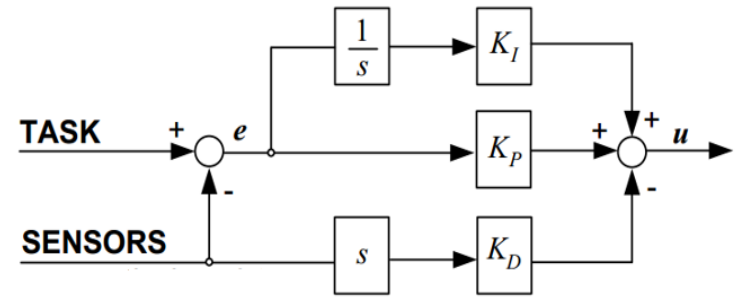

Fig. 3: The scheme of modified PID controller 
The output of PI-D controller can be represented in the following mathematical equation [29-32]:

$$
P I D(e)=k_{p} e(t)+k_{I} \int_{0}^{t} e(\tau) d \tau-k_{D} \frac{d}{d t} \text { state }
$$

where

$e(t)=$ desired state $(\mathrm{t})-$ measured state $(\mathrm{t})$

$k_{I}, k_{P}$ and $k_{D}$ are represent integral, proportional, and derivative gains respectively. The $\frac{d}{d t}$ state represents the derivative of state measured by sensor.

The desired control signals can be generated using equation 5 as follows:

$$
U_{j}=k_{p_{h}} \boldsymbol{e}_{\boldsymbol{h}}+k_{I_{h}} \int \boldsymbol{e}_{\boldsymbol{h}}-k_{D_{h}} \dot{h}_{\text {meas }}+m g
$$

where $j=[1, \ldots, 4], h=[z, \phi, \theta, \psi]$ and $e_{h}$ is the error signal, i.e. the difference between desired and measured states $\left(e_{h}=h_{\text {des }}-h_{\text {meas }}\right)$, where $h_{\text {des }}$ and $h_{\text {meas }}$ are represent the desired and measured values for the variable which we want to calculate control signal for it. The control signal $U 1$ is used to control the altitude of quadcopter while $U 2, U 3$, and $U 4$ are the calculated control signals that are used to control the three angles $\phi, \theta$, and $\psi$ of quadcopter. The variable $\mathrm{m}$ is represent the quadcopter mass and variable $g=9.81 \mathrm{~m} / \mathrm{s}^{2}$ is the earth gravity.

As we mentioned earlier, the real quadcopter has six degree of freedom $x, y, z, \phi, \theta$, and $\psi$ (three linear degree and the other is angular degree). The states $\mathrm{z}$ and $\psi$ are isolated while the other states $(\mathrm{x}, \mathrm{y}, \phi$, and $\theta$ ) are coupled and dependable because the quadcopter can't move towered $x$ and $y$ axis without change the value of $\phi$ and $\theta$. Therefore, the real quadcopter can perform 6-Degree of freedom through four control signals $\left(\mathrm{U}_{1}, \mathrm{U}_{2}, \mathrm{U}_{3}\right.$, and $\mathrm{U}_{4}$ ) generated by PI-D controller through desired $\mathrm{x}, \mathrm{y}, \mathrm{z}$, and $\psi$. In the simulation environment, the desired $\phi$, and $\theta$ are indirectly calculated by equations 10 and 11 through acceleration of desired $\mathrm{x}, \mathrm{y}$, and $\mathrm{z}$. The desired acceleration of $\mathrm{x}, \mathrm{y}$, and $\mathrm{z}$ can be calculated by using the following equations [27]:

$$
\begin{gathered}
\ddot{x}_{d e s}=\left(k_{p_{x}} e_{x}+k_{I_{x}} \int e_{x}-k_{D_{x}} \dot{x}_{\text {meas }}\right) / m \\
\ddot{y}_{d e s}=\left(k_{p_{y}} e_{y}+k_{I_{y}} \int e_{y}-k_{D_{y}} \dot{y}_{\text {meas }}\right) / m \\
\ddot{z}_{d e s}=\left(k_{p_{z}} e_{z}+k_{I_{z}} \int e_{z}-k_{D_{z}} \dot{z}_{\text {meas }}-m g\right) / m
\end{gathered}
$$

The following equations are used to calculate the desired $\phi$, and $\theta$ through desired acceleration of $x, y$, and $z$ which are calculated by equations 7,8 , and 9 [27].

$$
\phi_{\text {des }}=\sin ^{-1}\left(\frac{\ddot{x}_{\text {des }} s_{\psi}-\ddot{y}_{\text {des }} c_{\psi}}{\sqrt{\ddot{x}_{\text {des }}^{2}+\ddot{y}_{\text {des }}^{2}+\left(\ddot{z}_{\text {des }}+g\right)^{2}}}\right)
$$

$$
\Theta_{\text {des }}=\tan ^{-1}\left(\frac{\ddot{x}_{d e s} c_{\psi}-\ddot{y}_{d e s} s_{\psi}}{\ddot{z}_{d e s}+g}\right)
$$

The control block diagram of quadcopter dynamic model with PI-D controller can be shown in Fig. 4 [27].

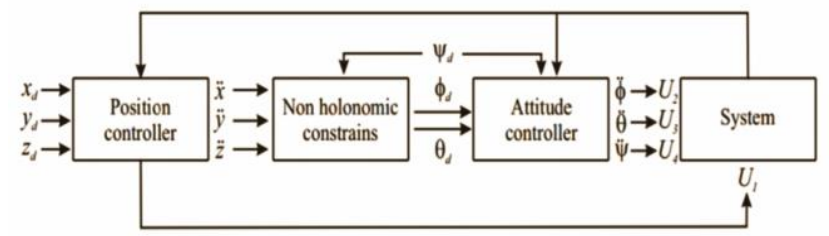

Fig. 4: The dynamic model block diagram of quadcopter with PI-D controller

\section{UNSCENTED KALMAN FILTER (UKF)}

The Unscented Kalman Filter (UKF) is one of the most reliable modern nonlinear filters that can be used with high nonlinearity systems in real time operation. Most of the previous filters such as KF and EKF use the linearization process through approximation Jacobian matrix to convert nonlinear system to linear one and then estimate accurate states of this system. The linearization process mean approximation in calculations. Also, linearization process has limited to operate with nonlinear systems and may push them out of control if high nonlinearity of system is occurring. The UKF is designed to operate very well with high nonlinearity systems. Two important process are used by UKF, the first one is called Sigma Points (SP) that the filter is generate a finite number of sigma points when applied to measured data that describes the system accurately. The second process is called Unscented Transformation (UT) which used to generate the sigma points from measured system data so the UKF does not need to linearization process. These two operations are designed to operate very well with high nonlinearity systems and provide high accuracy estimated states of systems. Please, for more details see [33-37].

The UKF predicts the states of the last estimated states by the following equation:

$$
\tilde{X}_{k+1 \mid k}=\phi_{k+1 \mid k}\left[X_{k \mid k}\right]+G_{k}
$$

The discrete predicted states $\tilde{X}_{k+1 \mid k}$ at $\mathrm{k}+1$ are updated with the measurement information by the following equation:

$$
\hat{X}_{k+1 \mid k}=\mathrm{H}_{k+1 \mid k}\left[\tilde{X}_{k+1 \mid k}\right]+V_{k+1}
$$

where $\phi_{k+1 \mid k}$ is the discrete state transition matrix from $\mathrm{k}$ to $\mathrm{k}+1, X_{k \mid k}$ is estimated states at $\mathrm{k}, G_{k}$ is the process noise vector at $\mathrm{k}, \hat{X}_{k+1}$ is the estimated state vector at $\mathrm{k}+1$, $V_{k+1}$ is the measurement noise vector, $\mathrm{H}_{k+1 \mid k}$ is the observation matrix. The state transition matrix is linear and derived from dynamic model acceleration equations of the quadcopter. Only the observation matrix $\mathrm{H}_{k+1 \mid k}$ contains nonlinear equations and it is calculated by the following equation: 


$$
\mathrm{H}_{k+1 \mid k}=\sum_{i=0}^{2 * n} W_{i} * H\left(X_{i, k+1 \mid k}^{\tilde{x}}\right)+X_{i, k+1}^{V}
$$

where $W_{i}$ are the weights, $X_{i, k+1 \mid k}^{\tilde{x}}$ are the sigma points which describe the predicted states $\tilde{X}_{k+1 \mid k}, X_{i, k+1}^{V}$ are the sigma points describing the measurement noise [33-36].

The number of sigma points depends on the dimensionality of the system. The general formula to calculate the number of sigma points is $2 n+1$, where $n$ is the dimension of the system.

The following equations are used to calculate the sigma points for the predicted states $\tilde{X}_{k+1 \mid k}[33,35]$ :

$$
\begin{gathered}
X_{0}=\overline{\tilde{X}} \\
X_{i}=\overline{\tilde{X}}+\left(\sqrt{(n+\lambda) p_{\tilde{x} \tilde{x}}}\right)_{i} \quad \mathrm{i}=1, \ldots \ldots, \mathrm{n} \\
X_{i+n}=\overline{\tilde{X}}+\left(\sqrt{(n+\lambda) p_{\tilde{x} \tilde{x}}}\right)_{i} \quad \mathrm{i}=\mathrm{n}+1, \ldots \ldots, 2 \mathrm{n}
\end{gathered}
$$

where $\overline{\tilde{X}}$ is the mean of the predicted states $\tilde{X}_{k+1 \mid k}, \lambda$ is the scaling factor which tells how far from the mean we should choose our sigma points, $p_{\tilde{x} \tilde{x}}$ is the covariance matrix of predicted states, $\left(\sqrt{(n+\lambda) p_{\tilde{x} \tilde{x}}}\right)_{i}$ is the $\mathrm{i}$-th column of the square root weighted covariance [33].

\section{LINEAR AND NONLINEAR ESTIMATION STATES USING UKF FILTER}

This section shows the explanation methodology of our proposed algorithm. The Matlab simulink version R2018a are used to simulate the overall proposed system (dynamic model of Quadcopter as well as the proposed estimation filter). All simulation processes are done on laptop with the following specifications: CPU core i5 $2.5 \mathrm{GHz}, 8 \mathrm{~Gb}$ of RAM, 512GB SSD hard drive and Windows 1064 bit. Fig. 5 shows simulation block diagram of overall proposed system which will use it to perform all necessary tests to know the effect of the proposed algorithm on the performance of the quadcopter. The dynamic model block diagram of quadcopter can be shown in Fig. 5 in addition to the estimation filter block and added white noise block.

In the first block, position controller, the linear position $\mathrm{x}, \mathrm{y}$, and $\mathrm{z}$ is controlled by using the equations $6,7,8$, and 9 in terms of acceleration these states. Also, in the first block, the control signal $\mathrm{U}_{1}$ is generated using desired acceleration altitude equation (equation 9). In the non-holonomic constraints block, the desired Roll $\left(\phi_{\text {des }}\right)$ and Pitch $\left(\Theta_{\text {des }}\right)$ angles are calculated with respect to the desired linear position $\left(X_{\text {des }}\right.$ and $Y_{\text {des }}$ ) using equations (10, and 11). In the angular position controller block, the equation (6) are performed for each angular states $(\phi, \theta$, and $\psi)$ to generate the control signals $U 2, U 3$, and $U 4$ which are used to control the angular position of quadcopter. Note that the force $g$ become zero in equation 6 when calculating $U 2, U 3$, and $U 4$ because $g$ has an effect perpendicular to the surface of the earth and effect only on altitude of quadcopter. The control signals $(U 1, U 2, U 3$, and $U 4)$ are feeds to the plant block were dynamic equations 1 and 2 are included to measure linear and angular states of quadcopter.

The white noise was added to the angular location $(\phi, \theta$, and $\psi$ ) to generate noisy angular position and make the simulations realistic. So, to decrease the effect of noise and estimate accurate angular states, the UKF is used to perform this task. Usually, the linear position ( $\mathrm{x}$, and $\mathrm{y}$ ) of quadcopter is measured by accelerometer sensor. The accumulation addition method is used by accelerometer sensor to measure the linear position at each next point [37]. Therefore, accumulated white noise is added to the measured linear position to get noisy linear position as shown in Fig. 5. Accurate linear states are estimated by the second UKF block added to the simulation blocks from noisy linear measured states.

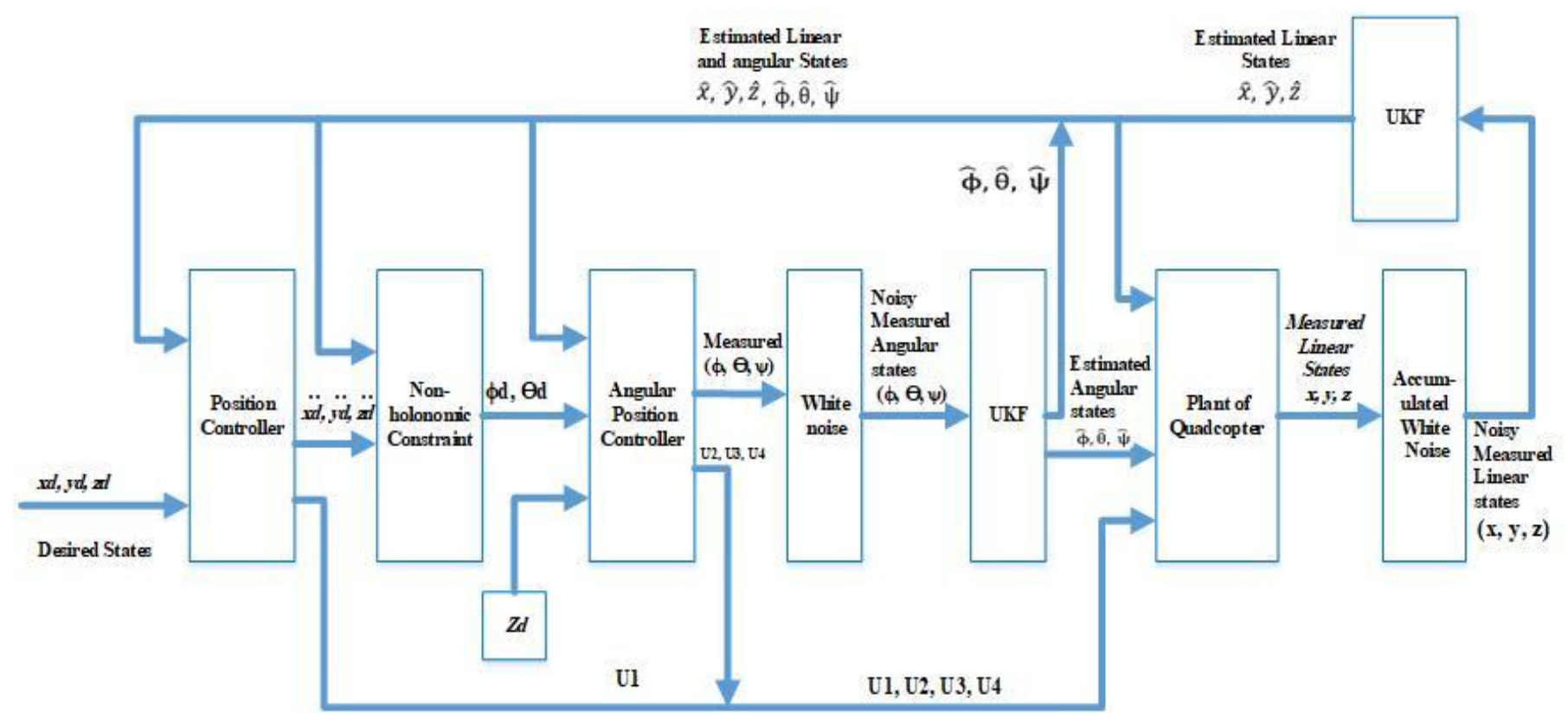

Fig. 5: The estimation and control block diagram of the quadrotor 


\section{RESULTS AND DISCUSSION}

The block diagram in Fig. 5 is performed in matlab simulink which represent the proposed algorithm and do all the required tests. Also the state transition functions (equation 3) and measurements functions (equation 4) are performed in Matlab file, so the UKF block can use them to perform his algorithm. Firstly, the dynamic model with modified PID (PI-D) controller is performed alone to get better tuned controller gains $\left(\mathrm{K}_{\mathrm{P}}, \mathrm{K}_{\mathrm{I}}\right.$, and $\left.\mathrm{K}_{\mathrm{D}}\right)$ for better response. After tuned all the gains of PI-D controller for all states, the square path for 200 seconds is used as a desired path for $\mathrm{X}$ and $\mathrm{Y}$ states while 10 meter is used as a desired altitude for $\mathrm{Z}$ state. This path was chosen because it is similar to many paths of quadcopter in the real world. Figure 6 shows the square desired path and the response of quadcopter using PI-D controller with and without UKF (UKF is used to estimate accurate linear and nonlinear states of quadcopter from noisy one).

The blue line in Fig. 6 is represent the desired path while the red and orange lines are representing the response of quadcopter with and without filter respectively. The zooming picture in Fig. 6c the noise is greatly decreased from measured linear and nonlinear states. Therefore, the response of quadcopter with UKF become smooth with very simple fluctuations (Red line). While the response of quadcopter without filter have much fluctuations and this lead to decrease the stability and performance of quadcopter through flight. The UKF is help to increase the efficiency of quadcopter control system and increase the stability and performance of quadcopter.

Figs. 7 and 8 shows the response of roll (Phi) and pitch (Theta) angles respectively without and with UKF. The response of angles with the filter are less fluctuations (the filter help to decrease the frequency of angle change). Also, the filer decrease peck to peck of angle change and this lead to decrease the vibration of the quadcopter and increase its stability.

The simulation speed of the motors can be calculated from equations 15 to 18 and converting this speeds to reality by using the transfer function shown in equation 19 [38]. The calculated real speeds of motors can be used as an indication that the simulations that have been performed are close to reality and there are no unrealistic values.

$$
\begin{aligned}
& w_{1}=\operatorname{sqrt}(u(1) /(4 * k)-u(3) /(2 * k * l)-u(4) /(4 * d)) \\
& w_{2}=\operatorname{sqrt}(u(1) /(4 * k)-u(2) /(2 * k * l)+u(4) /(4 * d)) \\
& w_{3}=\operatorname{sqrt}(u(1) /(4 * k)+u(3) /(2 * k * l)-u(4) /(4 * d)) \\
& w_{4}=\operatorname{sqrt}(u(1) /(4 * k)+u(2) /(2 * k * l)+u(4) /(4 * d))
\end{aligned}
$$

Also, these calculated speeds are transformed to the real quadcopter's speed motors using transfer function in equation 19 [38].

$$
T . F=\frac{0.936}{178 s+1}
$$

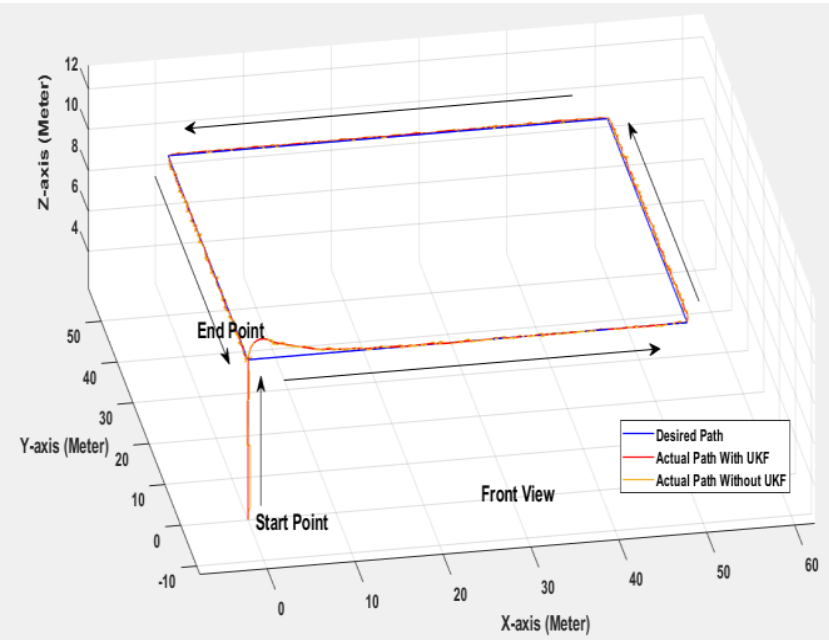

(a)

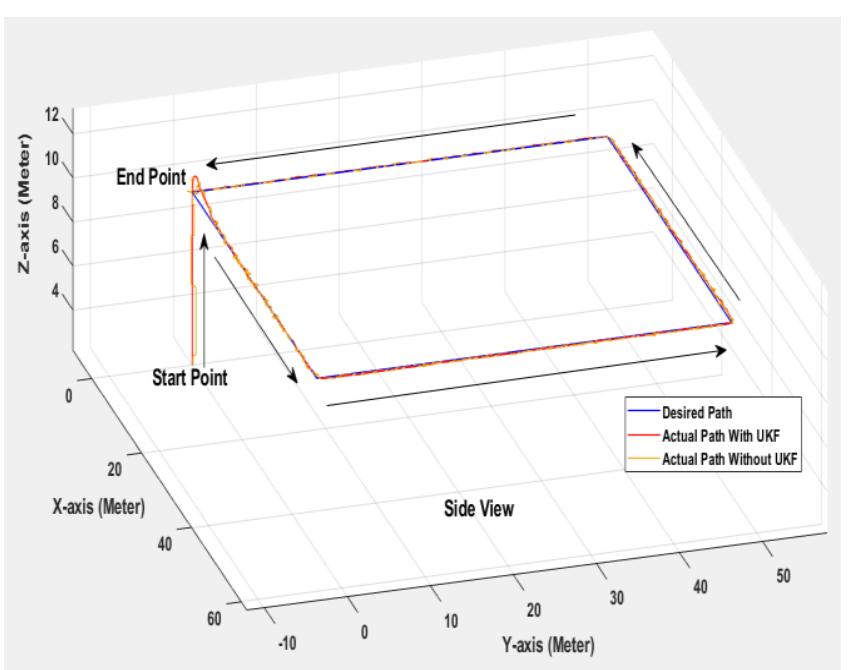

(b)

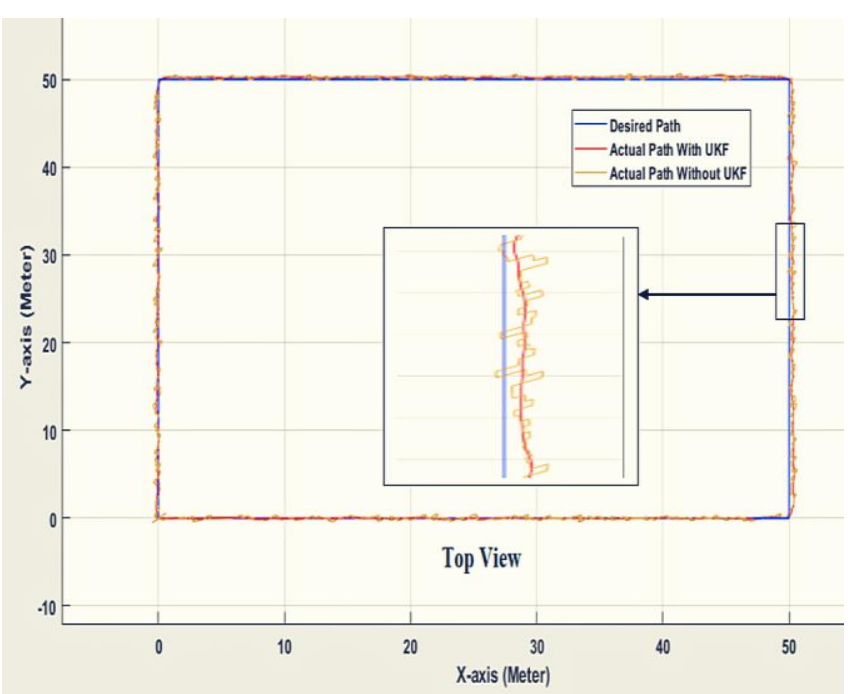

(c)

Fig. 6: The response of quadcopter with and without UKF 


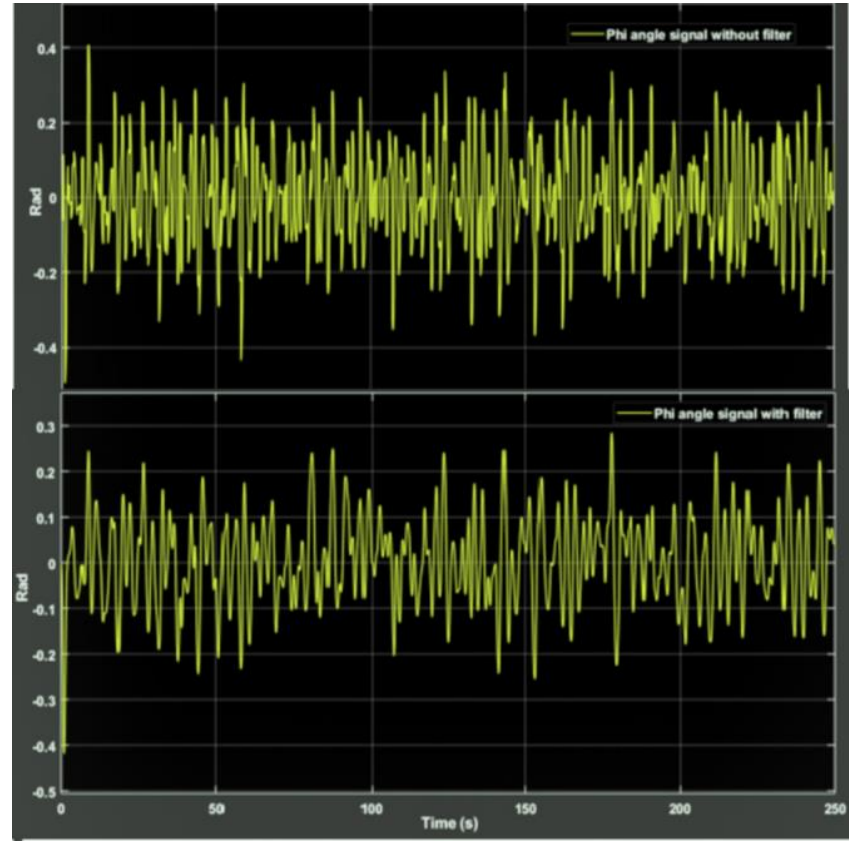

Fig. 7: The response of Roll (Phi) angle without and with UKF

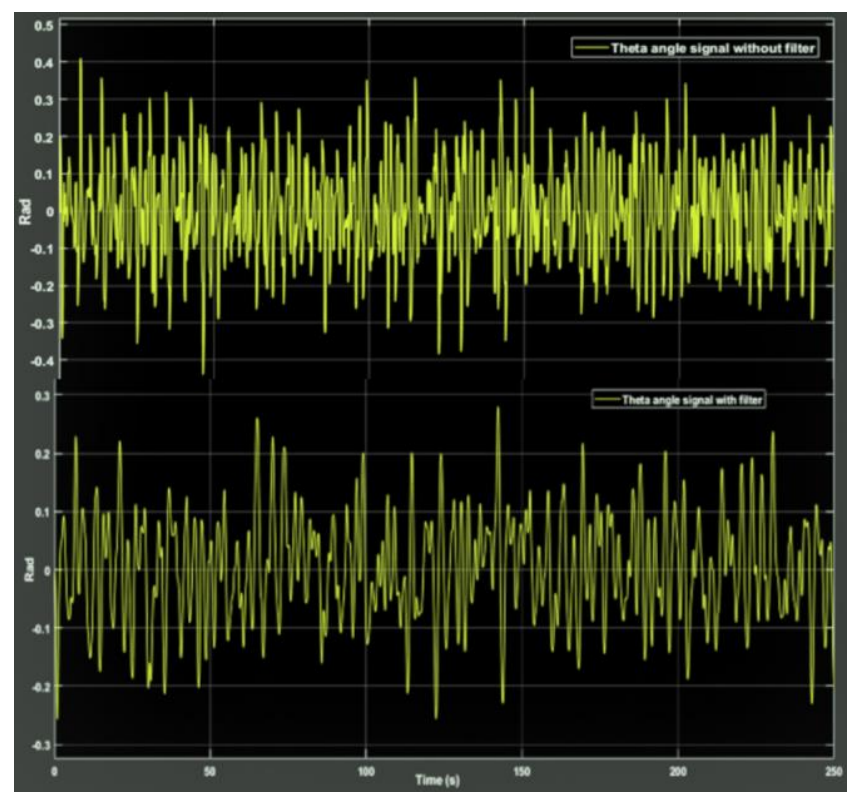

Fig. 8: The response of Pitch (Theta) angle without and with UKF

Fig. 9 shows the difference between the speed of the quadcopter's motors (rounds per minute) without and with filter while flying to follow the desired path shown in Fig. 6. The fluctuation in the speed of motors is very large (from 0 to $400 \mathrm{rpm}$ for the first 10 second of simulation) at beginning when the quadcopter takeoff from the ground to reach the desired altitude. After 10 second of time simulation, the quadcopter reach to desired altitude and start move toward $\mathrm{x}$ and $y$ axis. So, simple fluctuation is needed in speed of motors to change Roll and Theta angles and give ability to quadcopter move toward the desired path. After 10 second of time simulation, it is very clear from Fig. 9 that the fluctuation (peck to peck) of speed of motors with filter (233.3rpm to $235.8 \mathrm{rpm}$ ) is less than the speed without filter (233rpm to $247 \mathrm{rpm}$ ). The large fluctuation in the speed of motors caused by the noise generated by quadcopter sensors (Gyroscope and Accelerometer sensors).

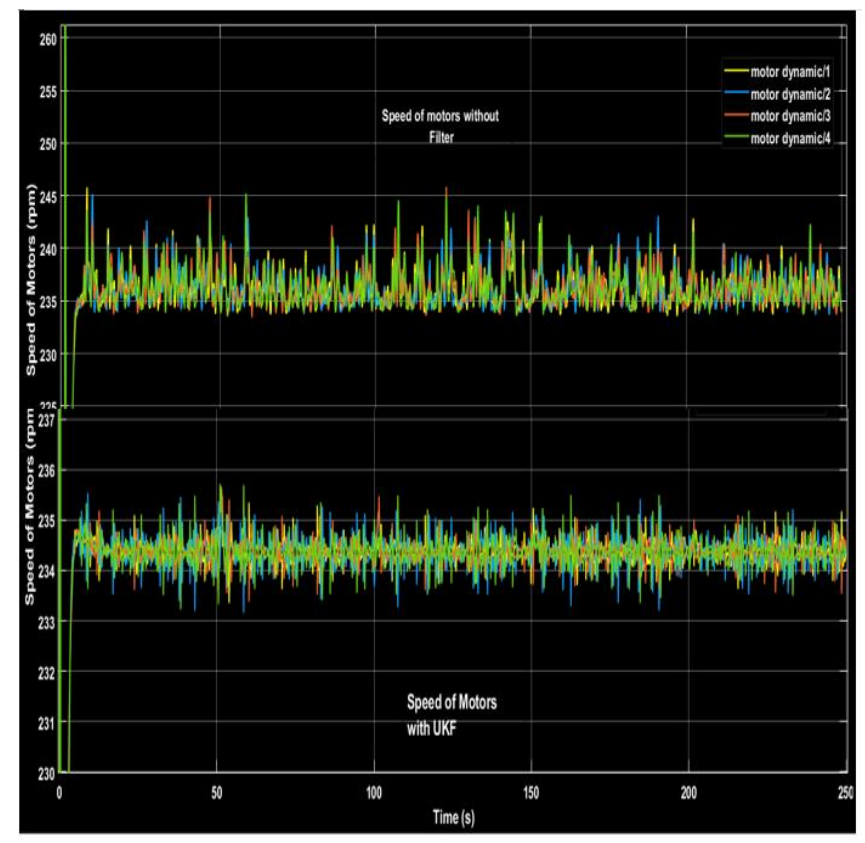

Fig. 9: The difference between the speed of motors without and with Filter

The large fluctuation in speed of motors lead to large vibration in the body of quadcopter through flight and this will decrease the stability and performance of quadcopter. The fluctuation in speed of motors is decreased after using the filter and this lead to increase the stability and performance of quadcopter.

Fig. 9 shows that the speed of motors $(0 \mathrm{rpm}(\mathrm{min})$ to $400 \mathrm{rpm}(\max ))$ is in the normal range of real world and this mean that there is no unrealistic parameter in our simulation.

\section{CONCLUSIONS}

The stability and performance of quadcopter are the important parameter that most researcher try to improve it through different algorithms. The noise generated by quadcopter's sensors is the most important issues that decrease the stability and performance of quadcopter. The proposed method in this paper is to reduce the effect of noise on both linear and angular states of quadcopter simultaneously. UKF was used with proposed method in this paper to reduce the noise effect and improve the performance of the quadcopter. The results showed that the proposed method in this paper is very efficient to decrease the noise from measured linear and angular states of quadcopter. The movement of quadcopter become more smooth comparing with the movement before applying proposed method. Decreasing the noise from measured states lead to decrease the fluctuations in the movements of quadcopter and increase its two important parameters, stability and performance. 


\section{CONFLICT OF INTEREST}

The authors have no conflict of relevant interest to this article.

\section{REFERENCES}

[1] MD. R. SOVON, "A thesis on Quadcopter", Thesis submitted to the university of Northwestern Poly technical, China, 2017.

[2] Syed O. F., "Building Smart Drones with ESP8266 and Arduino," Book published by Packt, Mumbai, India, 2018.

[3] Jose J., Karla A., Gerardo I., and Juvenal R., "Comparison of PD, PID and Sliding-Mode Position Controllers for V-tail Quadcopter Stability", IEEE Access Journal, Volume 6, pp. 38086 - 38096, 28 June 2018.

[4] Sevkuthan K., and Migdat H., "Control and Estimation of a Quadcopter Dynamical Model", Periodicals of Engineering and Natural Sciences Journal, Vol.6, No.1, pp. 63-75, March 2018.

[5] Pengcheng W., Zhihong M., Zhenwei C., Jinchuan Z., and Yong Z, "Dynamics Modelling and Linear Control of Quadcopter", IEEE International Conference on Advanced Mechatronic Systems, Melbourne, Australia, November 30 - December 3, 2016.

[6] Hannes B., "Quadcopter Control using Android-based Sensing", Master thesis, Lund University, Department of Automatic Control, 2013.

[7] Endrowednes K., Ioan T., Radu T., and Dan C., "Aspects Regarding Fly Control of Quadcopter", Recent Innovations in Mechatronics Journal (RIiM), Vol. 3, No. 1-2, (2016).

[8] Johan J., "Autonomous Localization and Tracking for UAVs using Kalman Filtering”, Master thesis, Norwegian University of Science and Technology, Faculty of Information Technology, Mathematics and Electrical Engineering, July, 2014.

[9] M. R. Mosavi, and I. Emam G., "De-noising of GPS Receivers Positioning Data Using Wavelet Transform and Bilateral Filtering", Springer, Wireless Personal Communications Journal, pp. 2295-2312, New York, 2012.

[10] J. A. A. S. Somasiri, D. P. Chandima, and A. G. B. P Jayasekara, "Extended Kalman Filter Based Autonomous Flying System for Quadcopters", IEEE, 2nd International Conference on Electrical Engineering (EECon), Colombo, Sri Lanka, pp. 130137, 28 Sep 2018.

[11] Kaiqiang F., Jie L., and Xiaoming Z., "A New Quaternion-Based Kalman Filter for Real-Time Attitude Estimation Using the Two-Step Geometrically-Intuitive Correction Algorithm”, MDPI, Sensors Journal, Vol. 17, pp. 1-22, 19 September 2017.

[12] A. Uma Mageswari, J. Joseph I., and R. Vinodha., "A Comparitive Study of Kalman Filter, Extended Kalman Filter And Unscented Kalman Filter For Harmonic Analysis of The Non-Stationary Signals", International
Journal of Scientific \& Engineering Research, Volume 3, Issue 7, July-2012.

[13] Dai H., Dai S., Cong Y., and Wu G.," Performance Comparison of EKF/UKF/CKF for the Tracking of Ballistic Target," Telecommunication, Computing, Electronics and Control Journal (TELKOMNIKA), Vol.10, No.7, pp. 1692-1699, November 2012.

[14] S. Konatowski, and A. T. Pieniężny, "A comparison of estimation accuracy by the use of KF, EKF \& UKF filters," WIT press, Transactions on Modelling and Simulation, Vol 46, P.P. 779-789, 2007.

[15] Stanislaw K., Piotr K., and Jan M., "Comparison of Estimation Accuracy of EKF, UKF AND PF Filters", WIT Transactions on Modelling and Simulation, Vol. 46, pp. 779-789, 2007.

[16] Jing C., Jerome C., Ali Z., Jorge D., and Jun Z., "Design of sliding mode observers for quadrotor pitch/roll angle estimation via IMU measurements", IEEE, Workshop on Research, Education and Development of Unmanned Aerial Systems (RED-UAS), Cancun, Mexico, November 23-25, 2015.

[17] Hector G., Fernando J., Jose M., and Felipe E., "UAV attitude estimation using Unscented Kalman Filter and TRIAD”, IEEE Transactions on Industrial Electronics, Vol. 59 , Issue 11 , pp. 4465 - 4474, Nov. 2012.

[18] Remus C., Xiaodong Z., Jacob C., and Jonathan M., "IMU Sensor Fault Diagnosis and Estimation for Quadrotor UAVs", Elsevier, International Federation of Automatic Control (IFAC) Journal, pp. 380-385, 2015.

[19] Zhenggao H., Guorong Z., Lei Z., and Dawang Z., "Fault Estimation for $N$ on linear Dynamic System Based", Circuits Syst Signal Process Journal, 30 April 2015.

[20] Aziz K., Ahmet E., and Emre K., "Model Derivation, Attitude Control and Kalman Filter Estimation of a Quadcopter", IEEE, International Conference on Electrical and Electronics Engineering, pp. 201-2014, 01 June 2017.

[21] Adrian B., Inna S., and Meyer N., "Attitude estimation for collision recovery of a quadcopter unmanned aerial vehicle", The International Journal of Robotics Research, Vol 38, Issue 10-11, pp. 1-21, 8 August 2019.

[22] Pengcheng W., Zhihong M., Zhenwei C., Jinchuan Z., and Yong Z., "Dynamics Modelling and Linear Control of Quadcopter", IEEE, Proceedings of the International Conference on Advanced Mechatronic Systems, Melbourne, Australia, pp. 498-503 November 30 December 3, 2016.

[23] Fernando H.C.T.E., De Silva A.T. A., De Zoysa M.D.C., Dilshan K.A.D.C, and Munasinghe S.R., "Modelling, Simulation Implementation pf a Quadrotor UAV", IEEE 8th International Conference on Industrial and Information Systems, ICIIS, pp. 207212, Sri Lanka, August, 2013.

[24] Sevkuthan K., and Migdat H., "Control and Estimation of a Quadcopter Dynamical Model", Periodicals of Engineering and Natural Sciences, Vol. 6, No. 1, pp. 63-75, March 2018. 
[25] M. Rich, "Model development, system identification, and control of a quadrotor helicopter", Graduate Theses and Dissertations, Electrical and Computer Engineering, Paper 12770, Iowa State University, 2012.

[26] Sumaila M., "Techniques for Quadcopter Modelling \& Design: A Review", Journal of Unmanned System Technology, Vol. 5, No. 3, pp. 66-75, 2017.

[27] Jose J. C., Karla A. C., Gerardo I. P., and Juvenal R., "Comparison of PD, PID and Sliding-Mode Position Controllers for V-tail Quadcopter Stability”, IEEE Access Journal, Vol. 4, 2018.

[28] Baqir N., Ammar A. A., "Outdoor \& Indoor Quadrotor Mission", Iraqi International for Electrical and Electronic Engineering Journal (IJEEE), The 3rd Scientific Conference for Researches at BASRAH, IRAQ, (15-16) JUNE 2020.

[29] Yaser A., and Aşkın M., "Modelling, Simulation and Implementation of Autonomous Unmanned Quadcopter", Journal of Machines, Technologies and Materials. 2018.

[30] Denis K., Zoran B., and Matija K., "Control Design for Unmanned Aerial Vehicles with Four Rotors", Journal of Interdisciplinary Description of Complex Systems14(2), page 236-245, march, 2016.

[31] Stephen A., Sun Yi, Wonchang C., and Dongchul S.,"Feedback Control of Quad-Rotors with a MatlabBased Simulator," American Journal of Applied Sciences, Vol. 13, Issue 6, pp. 779-793, USA, 2016.

[32] Bouzgou K., Bestaoui Y., Benchikh L., Ibari B., Ahmed F. Z., and Bouzgou K, "Dynamic Modeling, Simulation and PID Controller of Unmanned Aerial Vehicle UAV", IEEE, The Seventh International Conference on Innovative Computing Technology (INTECH), USA, 16-18 Aug 2017.

[33] T. Fiorenzani, C. Manes, G. Oriolo, and P. Peliti, "Comparative Study of Unscented Kalman Filter and Extended Kalman Filter for Position/Attitude Estimation in Unmanned Aerial Vehicles", Istituto di Analisi Sistemi ed Informatica, IASI-CNR, August, 2008.

[34] Mathieu S., and Denis G., "Comparison between the unscented Kalman filter and the extended Kalman filter for the position estimation module of an integrated navigation information system", IEEE Intelligent Vehicles Symposium Conference, Parma, Italy, pp. 831-835, 14-17 June, 2004.

[35] Eric A. Wan and Rudolph V., "The Unscented Kalman Filter for Nonlinear Estimation", In Proceedings of Symposium 2000 on Adaptive Systems for Signal rocessing, Communication and Control (AS-SPCC), IEEE Press. 2000.

[36] Simon J., Jeffrey U., and Hugh F., "A New Method for the Nonlinear Transformation of Means and Covariances in Filters and Estimators", IEEE Transactions on Automatic Control, Vol. 45, No. 3, MARCH 2000.

[37] Ehad A., Zoran S., and Akshya S., "A self-resetting method for reducing error accumulation in INS-based tracking", IEEE Conference, IEEE/ION Position,
Location and Navigation Symposium, USA, 08 July 2010.

[38] Heba talla M., "Dynamic Modeling and Control of a Quadrotor Using Linear and Nonlinear Approaches", Master thesis, American University in Cairo, School of Sciences and Engineering, 2014. 